\title{
BILDUNGEN MIT - VENLIG IM DÄNISCHEN UND IHRE POLNISCHEN ÄQUIVALENTE
}

\author{
ANDRZEJ SZUBERT
}

\section{Einführung}

Der folgende Artikel soll zur Erörterung der Bildungen mit -venlig in der dänischen Gegenwartssprache und ihrer polnischen Äquivalente beitragen und somit den Ausgangspunkt zu einer weiteren Untersuchung und Diskussion liefern. Bildungen mit -venlig sind ein recht neues Phänomen; der erste Beleg stammt aus dem Jahr 1954 (vgl. Riber Petersen, 1984: 523, Den Danske Ordbog online unter -venlig). "Ordbog over det danske Sprog" verzeichnet einige Beispiele wie z.B. bondevenlig, fremskridtsvenlig, forbrydervenlig, svenskvenlig, tyskvenlig mit der Anmerkung, dass venlig mit der Bedeutung 'venlig $(t)$ stemt' oft als zweites Glied in Komposita gebraucht wird. Die Struktur kommt parallel auch in anderen germanischen Sprachen wie z.B. eng. -friendly, dt. -freundlich, schw. -vänlig, no. -vennlig, holl. -vriendelijk usw. vor. Der Ausgangspunkt der Bildung ist natürlich das Adjektiv venlig, dessen Bedeutung in den Bildungen mit -venlig sich jedoch mit der Zeit verändert hat und heute von der Hauptbedeutung des Adjektivs venlig abweicht. Im Folgenden werden Bildungen mit -venlig in der dänischen Sprache mit ihren Interpretationsmustern dargestellt. Sie werden dabei mit den polnischen Äquivalenten kontrastiert, die in den meisten Fällen Nominalkonstruktionen sind.

Das Adjektiv venlig ist sehr populär und gebräuchlich im Dänischen und geht in den Wortschatz jeder Dänisch sprechenden Person ein. Nach "Den Danske Ordbog" (DDO) hat venlig die Bedeutung "behagelig og imødekommende over for andre" mit den Beispielen venlige mennesker, venlig dame, venlig mand. Dies veranschaulicht das Zitat: "Han er kendt som et venligt og besindigt menneske, der er behagelig at samarbejde med." JyP85. Außerdem gibt das DDO vier Unterbedeutungen an: 
1.a "som vidner om imødekommenhed, godt humør el. lign.": venlig betjening, venligt smil, venlige ord, venlige øjne, smile venligt, nikke venligt, venligt, men bestemt "Jan genkendte den venlige stemme med det samme" FamJour88

1.b "bruges formelagtigt som udtryk for høflighed i anmodninger, hilsener el.lign.": med venlig hilsen

„Medsend venligst frankeret svarkuvert” Hjemm92

1.c "behagelig at se på, opholde sig i el.lign. - om omgivelser og indretning

Se også -venlig"

"Lokalerne er nu sat flot i stand med lyse og venlige farver" SøbB191

1.d "(som sker) med hensigt om ikke at ændre i et selskabs forhold, fx i retning af opdeling og videresalg, uden selskabets egen indforståethed om opkøber eller opkøb af aktier"

Antonym fjendtlig

"Opkøbene ... var venlige overtagelser, som sikrede de nye ejere 100 procent kontrol med selskaberne" BørsNyhmag90."

Die Bedeutung 1.c weist auf das Lemma als Wortteil -venlig hin, wobei DDO das erste von den drei hier behandelten Wörterbüchern (die anderen sind "Retskrivningsordbogen" (RO) og "Ordbog over det danske Sprog" (ODS)) ist, das -venlig als ein Wortbildungselement verzeichnet. Dort steht nämlich, dass -venlig als Letztglied gebraucht wird, und dass der Gebrauch von 1954 bekannt ist. Es wurden folgende Bedeutungen angegeben: 'særlig indrettet til, egnet til eller god for', z.B. hudvenlig, dansevenlig. Das Zitat, das den Gebrauch illustriert, lautet:

"VOSS' glaskeramiske komfurer er køkkendivisionens frontløbere: avancerede, smarte og hurtige samt rengøringsvenlige" Samv-rek190.

Als grammatische Angabe steht dort "sidsteled" (Letztglied) und weist darauf hin, dass es als Glied in Komposita gebraucht wird.

"Ordbog over det danske Sprog" (online) gibt fünf Bedeutungen des Lexems venlig an, aber das Lemma -venlig kommt nicht vor, auch nicht im RO. In ODS wird erwähnt, dass venlig oft als Zweitglied in Zusammensetzungen gebraucht wird.

Im Deutschen ist der Gebrauch von entsprechenden Bildungen sehr ausgebreitet, und der "Duden online" betrachtet freundlich als Suffix, und die Bedeutungen des Lemma werden folgendermaßen erläutert:

"1. drückt in Bildungen mit Substantiven aus, dass die beschriebene Sache für jemanden, etwas günstig, angenehm, für etwas gut geeignet ist

Beispiel

magen-, reparatur-, verbraucherfreundlich

2. drückt in Bildungen mit Substantiven ein freundliches Entgegenkommen aus; wohlgesinnt gegenüber jemandem, etwas

Beispiel

kinder-, hundefreundlich" 
Als Lemma ist -venlig auch in Riber Petersens "Nye ord i dansk 1955-75" (1984) und Jarvads "Nye ord 1955-98" (1999) vorhanden, was in beiden Fällen mit Zitaten aus etlichen geschriebenen Quellen verdeutlicht wurde. Riber Petersen (1984: 523) gibt eine gut dokumentierte Beschreibung des bisher üblichen Gebrauchs von -venlig an und erwähnt, dass es mittlerweile auch einen neuen Gebrauch gibt: "om andet end personer, produktivt 2. led [...]". Sie schreibt auch, dass -venlig auf die gleiche Art und Weise gebraucht wird wie entsprechend -vennlig im Norwegischen und -vänlig im Schwedischen. In ihrem späteren Buch schreibt Jarvad (1999: 887f.) über das Lemma -venlig: "(produktivt andetled i udvidet brug om andet end personer) (1954)". Bei der letzten Beschreibung erwähnt Jarvad, dass entsprechende Bildungen auch im Englischen gebraucht werden: "Jf. tilsvarende brug af engelsk friendly." Das ist ein klares Signal, dass -venlig als Glied in Komposita betrachtet werden kann und dass es für jede dänischsprachige Person klar ist, dass es sich dabei um adjektivische Bildungen handelt. Nach DDO werden Bildungen mit -venlig Zusammensetzungen genannt. Die Forscher betrachten entsprechende Bildungen wie die mit -venlig in anderen germanischen Sprachen entweder als Komposita, wie Koniuszaniec (1989) im Falle des deutschen -freundlich, oder als Suffixoide, wie Ascoop (2005) mit schw. -vänlig og dt. -freundlich. Im Deutschen wird -freundlich als Suffixoid von u.a. Leuschner und Wante (2009) aufgefasst. Ob Bildungen mit -freundlich als Komposita oder Affixoidbildungen (vgl. Elsen, 2009: 328) zu betrachten sind, ist eine Diskussion, die im Rahmen dieses Artikels aus Platzgründen nicht erörtert werden kann. Elsen (2009) beschreibt ausführlich die Problematik hinsichtlich der Affixoide und präsentiert die Meinungen vieler Linguisten, die oft widersprüchlich und inkonsequent sind. Sie plädiert letztendlich für die Notwendigkeit der Einführung eines selbständigen Begriffs, der zwischen einem Derivat und Kompositum stehen würde - d.h. einer Affixoidbildung. Ohne auf die terminologische Diskussion einzugehen, schließe ich mich der Meinung von Elsen an, dass wir in solchen Fällen weder mit einem Kompositum noch einem Derivat zu tun haben. Meines Erachtens entsprechen sie doch eher den Komposita als den Derivaten, und deshalb würde ich sie Semikomposita und den Prozess Semikomposition nennen. Um nur einige der Argumente dafür vorzubringen, möchte ich betonen, dass in vielen Fällen Fugenelemente - wie bei den Komposita - gebraucht werden. Affixe sind nicht komplex, wie es Stämme sein können. Affixe unterliegen keiner weiteren wortbildungsmäßigen Analyse, was im Falle vieler Affixoide möglich ist (vgl. z.B. freundlich < Freund + -lich). Wenn man sagen kann, dass die Menge der Affixe einigermaßen begrenzt ist, ist die Anzahl der Stämme - und der potentiellen Semistämme - unbeschränkt. Über das lexikalisch-semantische Kriterium lässt sich folgendes sagen: Wenn man feststellen kann, dass ein Stamm eine lexikalische Bedeutung hat, und ein Affix zur Änderung der Wortklasse, Nuancierung oder Modifizierung der Bedeutung (vgl. dt. fahr +-en > Fahr + -er von einer Handlung zu derem Ausführer oder wie im Falle grün $>$ grün +- -lich) gebraucht werden kann, 
dann muss man ebenfalls konstatieren, dass die Situation solcher Elemente wie da. -venlig anders ist. Ihr Ausgangspunkt liegt in den Stämmen, und ihre Bedeutungen verändern sich mit der Zeit und weichen von den Grundbedeutungen der Lexeme ab, von denen sie stammen. Sie sind reihenbildend und in vielen Fällen werden sie zu selbständigen Lemmata in Wörterbüchern, obwohl dies nicht immer passiert. Dies gilt für da. -venlig, das ein selbständiges Lemma in DDO, bei Riber Petersen und Jarvad ist. Gleiches ist aber nicht der Fall in RO oder ODS. Es wird in ODS belegt mit Beispielen belegt wie:

arbejder-, bonde-, dyre-, folke-, forhandlings-, fremskridts-, konge-, menneske-, nabo-, samfunds-, socialistvenlig; forbryder-, freds-, svensk- tyskvenlig.

Sie sind nicht mehr freie Morpheme, sondern sie sind zu den gebundenen Morphemen geworden. In Hansen (1967 II: 454) werden ähnliche Bildungen aufgeführt, wobei der Autor -fceldig erwähnt, das er unter Zusammensetzungen behandelt und nennt ein Zusammensetzungsglied Glied. Weiter schreibt er: "At -fceldig ikke findes som selvstændigt adjektiv berettiger ikke opfattelsen af leddet som en afledningsendelse (sml. -formig ovf.)." Dies zeigt, dass das Problem doch recht kompliziert ist, und dass die Forscher verschiedene Auffassungen davon vertreten. Dies könnte der Ausgangspunkt für eine terminologische Diskussion sein, die aber nicht Thema dieses Artikels ist.

Das Ziel dieses Artikels ist es, Bildungen mit -venlig mit ihren strukturellen und semantischen Eigenschaften im Dänischen zu untersuchen und sie ihren polnischen Äquivalenten gegenüberzustellen. Es werden semantische Muster der Bildungen mit -venlig in der dänischen Sprache aufgestellt und die Art und Weise, auf die sie im Polnischen wiedergegeben werden, mit diesen kontrastiert. Nach Koniuszaniec (1989) sind Bildungen mit -freundlich im Deutschen sehr ausgebreitet. In der dänischen Sprachforschung wurde einer morphologisch-semantischen Analyse der Bildungen mit -venlig nicht viel Platz gewidmet.

Morphologisch gesehen sind Bildungen mit -venlig Strukturen, die wie Komposita aussehen, und sie werden auch so gebildet, abgesehen davon, dass ihre semantische Struktur von der determinativer Komposita abweicht. Man könnte sagen, dass die hier als Semikomposita genannten Bildungen, semantisch mehr den exozentrischen als den determinativen Komposita ähneln. Exozentrische Komposita werden nach einigen bestimmten Mustern ad hoc aus Stämmen gebildet, und sie verändern ihre Bedeutungen nicht. Dagegen ist es die semantische Struktur der Bedeutung der Bildung, die einem Wandel unterliegt. Semistämme (Erst- oder Letztglieder), die als Glieder mit Stämmen Semikomposita bilden, werden mit der Zeit im Verhältnis zu ihren Ausgangsstämmen semantisch modifiziert. Eine solche Bedeutungsmodifikation könnte man vielleicht "Bedeutungsverschleiß" nennen, - ein Phänomen wie es z.B. auch bei vaesen vorliegt, dessen Bedeutung sich etwa in Bildungen wie 
skolevcesen gänzlich verändert hat. Allmählich tauchen die hier Semmistämme genannten Elemente in den Nachschlagwerken als selbständige Lemmata und produktive Elemente auf, deren Bedeutung vom Ausgangslemma abweicht. Hinsichtlich der Fugenelemente ist das natürlich ein Argument in Richtung Komposition und nicht Derivation, und daher auch nicht Affixoidierung. Es ist das Erstglied, dass für das Fugenelement ausschlaggebend ist, wie in z.B. kattevenlig, das immer der Gemination des Auslautskonsonanten unterliegt und das $e$ als Fugenelement aufweist, während z.B. forhandling immer eine -s-Fuge fordert, wie in forhandlingsbord. Es gibt drei Fugenelemente, die in den gefundenen Bildungen mit -venlig vorkommen, und zwar -Ø-, -e-, -s- und eventuell die Verdoppelung des Auslautskonsonanten. Die Bildungen können auch in einer negierten Form vorkommen. Jedoch geschieht dies nicht durch das Ansetzen eines negierenden Präfixes vor der Bildung mit -venlig, vgl. *umiljøvenlig, sondern durch eine Negation des Semistammes -venlig, vgl. miljøuvenlig. Die Bildungen mit -venlig können als Substantive nur mit dem Suffix -hed zu größeren Einheiten ausgebaut werden.

Das Material zu dieser Untersuchung stammt vor allem aus DDO, ODS, RO und Riber Petersens "Nye ord i dansk 1955-75" (1984) (PRP) sowie Jarvads "Nye ord 1955-98" (1999) (PJ). Riber Petersen (1985: 523) und Jarvad (1999: 887f.) beinhalten viele Zitate, die den Gebrauch von -venlig veranschaulichen. Die Beispiele werden hier nicht nach den einzelnen Quellen eingeordnet, sondern nach ihren Bedeutungen. Statistisch gesehen soll aber erwähnt werden, dass die meisten Belege mit 29 Lemmata in DDO vorliegen, wogegen entsprechend 20 in ODS, 11 in RO, 7 in PRP und 8 in PJ verzeichnet sind. Einige von ihnen kommen in mehr als einer Quelle vor, aber außer dem RO beinhalten die übrigen Nachschlagwerke Beispiele, die in den anderen nicht verzeichnet sind. Es gibt nur vier Beispiele, die in allen drei Wörterbüchern vorkommen, und nur børnevenlig ist in allen den genannten Quellen vorhanden. Das bedeutet natürlich nicht, dass andere Bildungen in der Sprache nicht vorkommen. Die Autoren der belegten Beispiele gehen davon aus, dass es sich hier um einen sehr produktiven Typ handelt, und wie es hinsichtlich der Komposita der Fall ist, ist es nicht möglich, aber auch nicht nötig, sie alle in die Wörterbücher aufzunehmen, abgesehen davon, dass viele von ihnen Gelegenheitsbildungen sind.

Es gibt meines Erachtens eine besondere Gruppe der Bildungen mit -venlig, und zwar mit der Struktur: Verb + -venlig. In den Nachschlagwerken wie ODS, DDO und RO gibt es kein Beipiel dieses Typs. Zwei Beisliele - lcesevenlig und gå-venlig werden von Riber Petersen (1984: 523) und Jarvad (1999: 887f.) erwähnt, aber nicht mit der Absicht sie morphologisch zu betrachten, sondern lexikographisch. Dieser Typ kann aber ebenfalls sehr produktiv sein.

Im Folgenden werden die Beispiele nach der morphologischen Struktur (Substantiv + -venlig und Verb + -venlig - andere Wortklassen wurden nicht gefunden) und danach nach ihrer semantischen Struktur eingeordnet. Wenn man die Bedeutun- 
gen der Bildungen mit -venlig, die in DDO angegeben sind, analysiert, so können sie in fünf Mengen eingeteilt werden. Dazu kommen Beispiele, die mit der Anmerkung "Dette ord er registreret i Den Danske Ordbog, foreløbig uden betydningsangivelse." versehen sind. Dies bedeutet nicht, dass sie nicht gebraucht werden. Wenn man nach ihnen im Internet sucht, kann man in der Tat Belege für ihren Gebrauch finden. Die Internetsuche zeigt, dass sie in drei orthographischen Variationen vorkommen, und zwar zusammengeschrieben, mit Bindestrich geschrieben oder getrennt. Allerdings ist nur die erste Variation in Übereinstimmung mit der dänischen orthographischen Norm, aber dies ist ein Phänomen, das nicht Gegenstand dieses Artikels sein soll. Bei Jarvad (1999) erscheint das Beispiel additionsvenlig, das im Internet nicht gefunden wurde, was davon zeugen würde, dass es eine Gelegenheitsbildung war, und sich in der dänischen Sprache nicht eingebürgert hat. In vielen Fällen treten Analogiebildungen auf, die weitaus spezifischer sind. Dies gilt für z.B. mavevenlig im Verhältnis zu tarmvenlig. Dieses Verhältnis zwischen den beiden Bezeichnungen wird so verstanden, dass wenn man sagt Jeg har ondt i maven nicht genau bezeichnet, welches Bauchorgan nun weh tut. Ob das der Darm, Magen oder ein anderes Organ ist, wir in der Aussage nicht klar. Der Mechanismus schafft auf jeden Fall den Ausgangspunkt für weitere Bildungen. Allein dies macht deutlich, wie produktiv der Typ ist. Die vorliegende Untersuchung erhebt keinesfalls den Anspruch erschöpfend zu sein, aber es wird gehofft, dass sie ein Beitrag zu weiteren Untersuchungen sein könnte.

\section{Bildungen des Typs Substantiv + -venlig}

Die Struktur Substantiv + -venlig wird als einzige in den Nachschlagwerken verzeichnet. Obwohl Bildungen des Typs Verb + -venlig auch in der Sprache gebraucht werden, gibt es keine Belege in den oben genannten Wörterbüchern. Jarvad (1999: 887f.) erwähnt zwar loesevenlig und gå-venlig ohne weiter auf ihren Typ einzugehen. Nach der Analyse der Bedeutungsangaben in DDO (dort wo es sie gibt, denn lediglich bei 13 von 25 Stichwörtern werden hier die Bedeutungen angegeben) wurden Bedeutungsmuster für diese Konstruktionen aufgestellt. Das kann so gedeutet werden, dass wenn eine Bedeutung von z.B. brugervenlig angegeben ist, kann man davon ausgehen, dass die Deutung von forbrugervenlig oder familievenlig ähnlich durchzuführen ist. Die Beispiele wurden in "Deutungsgruppen" nach der Bedeutungsangabe in DDO oder nach eigener Deutung des Autors aufgrund der Internetbelege gruppiert. Die folgenden Gruppen sind das Resultat der Analyse. Als Ausgangspunkt gilt die Struktur $\boldsymbol{X}$ er $\boldsymbol{Y}$-venlig, die die folgenden Auflösungsparaphrasen (Deutungsparaphrasen) ergeben hat. Einige der Beispiele können in mehreren Kategorien vorkommen, weil sie auch verschiedene Bedeutungsnuancen haben können (z.B. børnevenlig - 'ikke skadelig for børn, let at betjene af børn, som tager 
hensyn til børn (f.eks. mindre stole, osv.)`. Es lassen sich folgende Kategorien aufstellen:

\section{1) $X$ ist leicht anzuwenden/bedienen für $Y$}

Dies gilt für einen Gegenstand, eine Anlage u.ä. X, der/die von Personen leicht $\mathrm{zu}$ gebrauchen ist, z.B.

brugervenlig, forbrugervenlig

\section{2) $\mathrm{X}$ ist eingerichtet/geeignet für $\mathrm{Y}$}

Der Gegenstand X ist absichtlich so konstruiert, dass X an Y angepasst, für den Gebrauch von Y berechnet ist, wo Y Personen oder Handlungen umfasst, z.B.

barnevenlig, beboervenlig, blindevenlig, bordvenlig, brugsvenlig, børnevenlig, dansevenlig, døvevenlig, familievenlig, laservenlig, postvenlig, reolvenlig, servicevenlig

\section{3) X nimmt Rücksicht auf $Y$}

$\mathrm{X}$ ist ein Gegenstand, ein Vorschlag, eine Regel, der oder die Y berücksichtigt, respektiert, z.B.

budgetvenlig, danskvenlig, dyrevenlig, fuglevenlig, kattevenlig, lønmodtagervenlig, menneskevenlig

\section{4) $\mathrm{X}$ ist gegenüber $\mathrm{Y}$ entgegenkommend}

X kann ein Gesetz, ein Beschluss, eine Person oder Institution sein, die positiv eingestellt ist oder etwas positiv gegenüber $\mathrm{Y}$ formuliert. Y kann eine Person, Institution u.ä. sein, z.B.

atlanterpaktvenlig, arbejdervenlig, bondevenlig, erhvervsvenlig, folkevenlig, forbrydervenlig, forhandlingsvenlig, forsvarsvenlig, fredsvenlig, fremskridtsvenlig, gastevenlig, kongevenlig, kundevenlig, nabovenlig, nazivenlig, noestevenlig, publikumsvenlig, reformvenlig, regeringsvenlig, samfundsvenlig, tyskvenlig, økonomivenlig

$\mathrm{Zu}$ dieser Gruppe gehört auch handicapvenlig / handikapvenlig, in dem der Stamm handicap / handikap vorkommt. Allerdings wird bei Anwendung des Wortes auf Personen mit Behinderung hingewiesen, und nicht auf die Behinderung selbst. Das kann mit der sprachlichen "Bequemlichkeit" verbunden sein.

\section{5) $\mathrm{X}$ ist schonend für $\mathrm{Y}$}

$\mathrm{X}$ ist gebaut, funktioniert u.ä. auf die Art und Weise, dass es absichtlich schonend für $\mathrm{Y}$ ist. Dies kann als $\mathrm{X}$ ist gut für Y gedeutet werden, z.B. 
damefodsvenlig, energivenlig, fingervenlig, fodvenlig, hudvenlig, kropsvenlig, livsvenlig, mavevenlig, miljøvenlig, naturvenlig, numsevenlig, tarmvenlig, vejvenlig

\section{6) $\mathrm{X}$ lässt das Vorkommen von $\mathrm{Y}$ nicht zu}

$\mathrm{X}$ beschränkt oder lässt das Vorkommen von Y nicht zu, z.B.

allergivenlig, støjvenlig.

Unter den Beispielen ist kattevenlig, das außer der endozentrischen (direkten) auch eine exozentrische Bedeutung "venlig på en påtaget og (lidt for) indsmigrende måde" (DDO online) hat. Ob dansevenlig auch unter dieser Struktur aufgelistet werden soll, ist nicht klar, weil man nicht mit Sicherheit sagen kann, ob die Rede von einer Bildung, in der wir mit dem Substantiv dans und dem Fugen-e zu tun haben, oder mit einem Verbalstamm und Fugen-e. Das ist ein rein morphologisches Problem, weil die semantische Interpretation in beiden Fällen gleich ist.

\section{Bildungen des Typs Verb + -venlig}

Nur bei Riber Petersen (1984: 523) und Jarvad (1999: 887f.) kann man Beispiele von Bildungen mit der Struktur Verb + -venlig finden, es sind zwei: laesevenlig und gå-venlig. Es ist schwierig zu beurteilen, wie viele Bildungen des Typs Verb + -venlig gebraucht werden. Die Internetsuche hat nicht besonders überraschend ergeben, dass es solche Bildungen gibt, und dass sie oft gebraucht werden. Sie sind einfach zu bilden, weil sie immer mit der Fuge-e im Falle der Verbstämme, die eine Infinitivendung bekommen, und -Ø- bei den übrigen Verben (z.B. gåvenlig) gebildet werden. Die Anzahl der Verben in solchen Bildungen ist im Verhältnis zu den substantivischen Stämmen bedeutend geringer. Viele von ihnen kommen in drei orthografischen Formen - zusammengeschrieben, mit dem Bindestrich und getrennt als zwei Wörter - vor. Die Untersuchung hat ergeben, dass es bedeutende Quantitätsunterschiede zwischen den einzelnen Bildungen geben kann, z.B. hat loesevenlig 99600 Belege und huskevenlig 27. Zwischen ihnen liegen solche wie: rygevenlig (50, aber auch rygervenlig, das aus dem Substantiv und -venlig besteht, vgl. ryger + venlig, 128), spisevenlig (286), skrivevenlig (321), siddevenlig (1200), gåvenlig (1760, kommt in drei orthografischen Formen: gåvenlig, gå-venlig, gå venlig), kørevenlig (2400) (z.B. knallert). Dagegen hat die Suchung nach ståvenlig keine Ergebnisse gegeben, ungeachtet der Schreibform. Man könnte natürlich mehr Bildungen bilden und untersuchen, ob sie im Dänischen gebraucht werden, aber ihre semantische Struktur bleibt die gleiche. Semantisch werden sie interpretiert als:

$\mathrm{X}$ ist so konstruiert / gebaut / eingerichtet, dass $\mathrm{X}$ die Handlung $\mathrm{Y}$ leichter, angenehmer macht, z.B. 
gåvenlig, huskevenlig, kørevenlig, loesevenlig, rygevenlig (auch rygervenlig), siddevenlig, spisevenlig, skrivevenlig.

Obwohl die Bildungen des Typs Verb + -venlig in den Wörterbüchern nicht vertreten sind, bedeutet dies nicht, dass solche Konstruktionen von den Muttersprachlern und auch Ausländern nicht gebildet und gebraucht werden. Dies kann daraus hervorgehen, dass sie einfach zu bilden sind, und dass ihre Ausdrucksseite umfassend ist.

\section{Die polnischen Äquivalente der dänischen Bildungen mit -venlig}

Die Frage, wie man die dänischen Bildungen mit -venlig im Polnischen wiedergeben soll, ist nicht so übersichtlich zu beantworten, wie man zunächst glauben sollte. Morphologisch gesehen gibt es keine ähnlichen Bildungen im Polnischen, obwohl sie natürlich wiedergegeben werden können, selbst wenn sie manchmal etwas künstlich lauten mögen. Im Folgenden werden aufgrund der Beispiele einige Möglichkeiten ihrer Wiedergabe in der polnischen Sprache dargestellt. In einigen Fällen sind das Derivate, die den dänischen Bildungen entsprechen, wie z. B. gaestevenlig - gościnny, menneskevenlig - ludzki, kattevenlig - nieszczery (obwohl eine endozentrische Bedeutung auch als przyjazny kotom vorkommt), miljøvenlig - ekologiczny (aber auch przyjazny dla środowiska). Sie sind jedoch in den meisten Fällen alte und gut etablierte Wörter, die nicht unbedingt die dänischen Bildungen als Ursprungsquellen haben. Den neueren Äquivalenten liegen bereits die Bildungen mit -venlig zugrunde, und sie kommen als Nominalkonstruktionen mit analytischer Struktur vor.

Die größte Gruppe der Äquivalente weist die Strukturen auf:

1) $\mathbf{X}$ jest przyjazny dla $Y$ - ' $X$ ist freundlich gegenüber $Y$ ' ( $Y$ ist ein Substantiv im Genitiv), z.B.

X jest przyjazny dla klienta (Kunde), przedsiębiorcy (Unternehmer), rowerzystów (Radfahrer), nowoczesnych technologii (moderne Technologien), uzytkownika (Benutzer), konsumenta (Konsument), środowiska (Milieu), rodziny (Familie), pacjenta (Patient), ptaków (Vögel), skóry (Haut) usw.

Diese Struktur kommt aber in einigen Fällen auch als $\mathbf{X}$ jest przyjazny $\mathbf{Y}_{\text {DATIV }}$ (z.B. przyjazny środowisku).

Dies zeigt, dass die Polen in vielen Fällen wohl nach dem englischen Einfluss die oben genannte Struktur gebrauchen, obwohl die Bedeutung von przyjazny (freundlich) mit dem Bedeutungsumfang: "Rücksicht nehmen", "leicht / einfach zu gebrauchen / benutzen", "entgegenkommend / schonend sein", "speziell eingerichtet / gebaut sein“" usw. erweitert wurde. Dies ist ein Ausdruck davon, dass eine ziemlich 
einfache Bildung im Dänischen (bzw. im Deutschen) einer mehr komplexen Struktur in einer anderen Sprache entsprechen kann.

2) $X$ jest pozytywnie nastawiony do $Y$ - ' $X$ ist gegenüber $Y$ ' positiv eingestellt ( $Y$ ist im Polnischen ein Substantiv im Genitiv)

$\mathbf{X}$ jest pozytywnie nastawiony do negocjacji (Verhandlungen), NATO, planu (Plan), rządu (Regierung), tradycji (Tradition), Duńczyków (Dänen), postępu (Fortschritt) osv.

In den meisten Fällen decken die oben genannten Konstruktionen den Gebrauch der dänischen Bildungen mit -venlig. Die Bedeutung des Adjektivs przyjazny 'freundlich" hat sich mit der Zeit wie die Bedeutung des dänischen -venlig erweitert. Es begegnen polnische Äquivalente der dänischen Bildungen mit -venlig, die eine mehr komplexe Struktur aufweisen, wie z.B. brugervenlig - 'specjalnie skonstruowany, urządzony itd. dla użytkownika' (speziell konstruiert, eingerichtet usw. für den Benutzer). Als Prinzip kann gesagt werden, dass die dänischen Bildungen mit -venlig als nominale Konstruktionen wiedergegeben werden. In der ersten Gruppe ist die Rede von einer adjektivischen Präpositionalphrase, die der dänischen Bildung mit -venlig entspricht. Mit einer beschreibenden und komplexeren Wiedergabe haben wir es im Falle der zweiten Gruppe zu tun. Der Bedeutungsumfang ist doch mit der Mehrdeutigkeit des Lexems venlig belastet, das im Polnischen mehrere Äquivalente haben kann, wie u.a. uprzejmy, mily, przyjazny, przyjacielski, sprzyjajacy, korzystny, koleżeński, sympatyczny. Da venlig so viele Äquivalente im Polnischen haben kann, ist die Wiedergabe der dänischen Bildungen mit einer gewissen Unsicherheit belastet. Der Kontext ist hier entscheidend, jedoch hat die Untersuchung das Ergebnis gebracht, dass die Konstruktionen unter 1) in den meisten Fällen gebraucht werden, wo die Bedeutung von przyjazny um weitere Bedeutungen erweitert wurde, die dem dän. venlig bzw. dt. freundlich entsprechen. Wenn man nach Wiedergaben des eng. -friendly sucht, findet man z.B. eye-friendly - cieszacy oko ('das das Auge freut'), przyjemny dla oka ('angenehm für das Auge') oder user-friendly - tatwy w użyciu / obstudze ('leicht im Gebrauch, in der Bedienung'). Wie aus den Beispielen hervorgeht, ist es nicht einfach, ein bestimmtes Muster anzugeben.

\section{Zusammenfassung}

Die Bildungen mit -venlig im Dänischen (aber auch in anderen germanischen Sprachen) sind ein ziemlich neues Phänomen und zeigen die Entwicklung, dass das Lexem venlig, das die Bedeutungen von etwa " $\mathrm{X}$ tager hensyn til $\mathrm{Y}$ " und "X skaber ikke fare for Ys optræden eller begrænser den" repräsentiert, wobei die letztere im Gegensatz zu der Grundbedeutung steht. Dies zeugt davon, dass -venlig als ein Semistamm (bei den meisten Linguisten Affixoid) betrachtet werden kann. 
Einige der Bildungen mit -venlig (aber auch entsprechende Bildungen in anderen germanischen Sprachen) haben lexikalisierte, synthetische Äquivalente im Polnischen wie z.B. kattevenlig - nieszczery, geestevenlig - gościnny. In anderen Fällen müssen analytische nominale Konstruktionen gebildet werden. Es sind adjektivische oder partizipiale Präpositionalphrasen. Es war nicht die Absicht mit dem Artikel, alle Wiedergabemöglichkeiten der dänischen Bildungen mit -venlig im Polnischen zu zeigen, sondern vielmehr die Grundmuster anzugeben, in der die Bildungen wiedergegeben werden, wie z.B. przyjazny środowisku, sprzyjający środowisku, dobry dla środowiska, bezpieczny dla środowiska, niegroźny dla środowiska. Dies zeigt, wie schwierig es im Polnischen ist, ein einziges Äquivalent einer bestimmten Bildung mit -venlig anzugeben.

Es ist jedoch auch wichtig zu unterstreichen, dass selbst wenn es eine -venligKonstruktion in einer Sprache wie z.B. Dänisch gibt, bedeutet dies nicht, dass strukturmäßig äquivalente Konstruktionen in anderen, auch sehr verwandten Sprache vorkommen, weil es trotzdem Unterschiede im Gebrauch der Bildungen mit -venlig im Dänischen im Vergleich zu anderen germanischen Sprachen gibt.

\section{Literatur}

Ascoop, K. 2005. "Affixoidhungrig? Skitbra! Status und Gebrauch von Affixoiden im Deutschen und Schwedischen“. Germanistische Mitteilungen 62. 17-28.

Elsen, H. 2009. "Affixoide: Nur was benannt wird, kann auch verstanden werden". Deutsche Sprache 37. 316-333.

Hansen, Aa. 1967. Moderne dansk. Bind II. København: Det Danske Sprog- og Litteraturselskab. Grafisk forlag.

Jarvad, P. 1999. Nye ord. Ordbog over nye ord i dansk 1955-1998. København: Gyldendal. (verkürzt im Text zu PJ).

Koniuszaniec, G. 1989. „Beobachtungen zum Kompositionstyp Substantiv + “freundlich” im Vergleich mit dem Polnischen“. Studia Germanica Posnaniensia XVI. 163-168.

Leuschner, T. und E. Wante. 2009. "Personale Suffixoide im Deutschen und Niederländischen“. Germanistische Mitteilungen 70. 59-73.

Riber Petersen, P. 1984. Nye ord i dansk 1955-75. Dansk Sprogncevns skrifter 11. København: Gyldendal. (verkürzt im Text zu PRP).

\section{Internetquellen}

Den Danske Ordbog online http://ordnet.dk/ddo (11.07.2016) (hier abgekürzt DDO)

Ordbog over det danske Sprog online http://ordnet.dk/ods (11.07.2016) (hier abgekürzt ODS)

Retskrivnngsordbogen online http://www.dsn.dk/ (11.07.2016) (hier abgekürzt RO)

Duden online http://www.duden.de/woerterbuch (11.07.2016) 
